\title{
Propuesta de medidas para una gestión responsable de la diversidad en las organizaciones en el escenario covid y poscovid
}

\author{
Paul Parra-Moreno \\ Universidad Pontificia de Comillas \\ pparra@comillas.edu
}

Resumen. La gestión de la diversidad en las organizaciones se posiciona como estrategia necesaria para una exitosa respuesta frente a los cambios y desafíos derivados del escenario covid. Atender a la diversidad conlleva mejoras internas en el desempeño, en la productividad, en el clima laboral o en la búsqueda de soluciones más creativas y flexibles ante los problemas, además de mejoras externas en la consolidación de la marca y en la apertura a nuevos mercados. Analizamos los posibles beneficios, desafíos, dilemas y retos que, a raíz de la crisis generada por la covid-19, aparecen en los modelos de gestión responsable de la diversidad en las organizaciones. En este trabajo desarrollamos una discusión a partir de la entrevista realizada a la entidad española Fundación Once como ejemplo de buenas prácticas en la implementación de políticas inclusivas, y analizamos los datos extraídos resultado de la búsqueda bibliográfica con artículos firmados por personas $y / o$ entidades relevantes en el tema propuesto. Como resultado de las conclusiones principales, elaboramos una propuesta de medidas que desarrollar por las organizaciones para establecer y/o conservar una politica estratégica en la gestión responsable de la diversidad en el escenario coronavirus.

Palabras claveः coronavirus; corresponsabilidad; diversidad; gestión. 
Proposed measures for the Responsible MaNAgement of DiVersity in ORgANizATIONS DURING AND AFTER COVID-I9

Abstract. Diversity management in organizations is positioned as a necessary strategy in a successful response to the changes and challenges arising from COVID-19. Addressing diversity leads to internal improvements in performance, productivity, the work climate or the search for more creative and flexible solutions to problems, as well as external improvements in brand consolidation and opening up to new markets. We analyze the possible benefits, dilemmas and challenges that, in the wake of the crisis generated by COVID-19, appear in models for the responsible management of diversity in organizations. In this paper we discuss the Spanish entity Fundacion Once as an example of good practice in the implementation of inclusive policies, and we analyze the data extracted from a bibliographic search for articles authored by people and/or entities relevant in the proposed area. On the basis of the main conclusions that we found, we developed a series of measures that could be adopted by organizations to establish and/or maintain a strategic policy regarding the responsible management of diversity in during and after COVID-19.

Keywords: Coronavirus; Co-responsibility; Diversity; Management. 


\section{Introducción}

Cuando las organizaciones cuentan con un capital humano diverso en sus plantillas, no dejan de atender a la realidad social en la que están insertas. De esta manera, desarrollan un papel activo en la transformación de la sociedad, convirtiéndose en agentes de cambio, capaces de dar respuesta a las diferentes problemáticas y retos propios de sociedades líquidas y globales (Asociación Comisión Católica Española de Migración, ACCEM, 2020).

En la gestión responsable de la diversidad, las organizaciones desarrollan de forma proactiva un proceso de integración y adaptación de las diferencias culturales, diversidades de género, capacidades diferentes y otras, convirtiéndolas en potencial valor añadido, por una mejora sustancial y por una consolidación de las expectativas organizacionales, tanto internamente, en el sentido de pertenencia de los empleados, como externamente, resultando más competitivos en el mercado.

Para que este modelo de gestión sea eficaz y efectivo, es necesario que estas diversidades sean integradas en los sistemas económicos, de mercados o de productos. La diversidad de potenciales socios y clientes necesita de organizaciones que den respuesta a esta heterogeneidad (ACCEM, 2020).

Siguiendo el informe Gestión de la diversidad de la Fundación CEPAIM (2013), se apunta a la necesidad de aplicar tres pasos básicos en una gestión responsable de la diversidad.

En primer lugar, se habla de diagnosticar y analizar la diversidad que reconocemos en el entorno, tanto interno como externo. En segundo lugar, se necesita trabajar por la consecución de una comunicación tanto interna como externa que sea efectiva, fluida y eficaz en la transmisión de las medidas adoptadas por parte de todas las personas implicadas en la entidad. En tercer lugar, es importante integrar la diversidad diagnosticada en los fines y estrategias de las organizaciones, así como en la cultura y los valores de estas.

Asimismo, siguiendo el informe Empresas que SUMAN. Reto 2030, entendemos como la gestión responsable de la diversidad es una oportunidad de mejora de las organizaciones insertas en sociedades plurales, convirtiéndose en agentes de cambio fundamentales en el desarrollo de «sociedades más inclusivas, cohesionadas y sostenibles» (ACCEM, 2020: 3), objetivos alineados con los objetivos de desarrollo sostenible de la Agenda 2030.

Además del valor social y la corresponsabilidad que supone la implementación y desarrollo de estos planes, la gestión de la diversidad en las organizaciones ayuda a atraer y retener el talento, por encima de estereotipos y limitaciones. 
Esto supone para el trabajador una mayor motivación, y ofrece nuevas oportunidades de negocio pues la organización será capaz de moverse en un entorno diverso.

Para alcanzar en los lugares de trabajo ambientes inclusivos y diversos donde se alcance la igualdad de oportunidades en el mercado laboral, es necesario conseguir la eliminación de la discriminación. Diversidad e inclusión es esencial para estimular nuevas ideas e innovadoras, con beneficios transversales en la sociedad. La comisión de la UE afirma que hay una correlación entre inclusión y el retorno de bienes, productividad, innovación (Comisión Europea, 2020ः 9).

Integrar capital humano diverso en las plantillas aporta diferentes modos de ver $y$ hacer, con diversas perspectivas y visiones sobre el mundo, las cuales permiten ampliar el abanico de consideraciones y toma de decisiones, de manera que la empresa más solvente en trabajo interno se convierte en más competitiva, lo que a su vez permite la expansión a nuevos mercados y clientes (Casanova, 2006).

Para conseguir esta diversidad en las organizaciones es necesario convertirla en objetivo estratégico que afecte a todos los escalafones de la organización, como en la formación o en los procesos de contratación (Velasco, 2020).

Para ello, necesitamos reconocer la heterogeneidad que se da en las sociedades contemporáneas y quienes la forman. Esta gran diversidad demográfica, experiencial o cognitiva, debería estar integrada y representada en los stakeholders de la organización.

El reconocimiento de las diferentes, tanto sociodemográficas (edad, género, nacionalidad, formación, antigüedad, función, nivel educativo...) como de capital humano (conocimientos, esquemas cognitivos, experiencias, valores...) que hacen a las personas únicas y a los grupos heterogéneos (ACCEM, 2020: 5).

Con la crisis derivada de la covid-19, que a nivel mundial ha desestabilizado el normal funcionamiento de sistemas sanitarios, económicos y sociales, nos planteamos de qué maneras se ve afectada la implantación y desarrollo de planes y políticas de la gestión de la diversidad en las organizaciones como modelo responsable de gestión de los recursos humanos.

Nos preguntamos en qué medida las políticas de diversidad existentes en las organizaciones han sido y son útiles para gestionar mejor al personal en esta crisis; durante estos últimos meses de alarma, qué medidas se han desarrollado según las situaciones/características de cada empleado; cuáles son los principales desafíos y cambios para una gestión responsable de la diversidad a partir de la crisis generada por la covid-19, y cuáles han sido las principales lecciones aprendidas durante estos meses de alarma. 


\section{Metodología}

Con el fin de abordar el estado de la cuestión, hemos explorado los más recientes informes e investigaciones publicadas en relación con los cambios y retos en la implementación y desarrollo de planes en gestión de la diversidad en las organizaciones.

Durante la elaboración, recogida y análisis de la bibliografía utilizada, así como de la entrevista realizada, hemos intentado conocer cuáles han sido los principales beneficios, problemáticas, barreras, desafíos y soluciones que han surgido a raíz de la crisis derivada de la covid-19 en relación con los planes de gestión de diversidad, $y$, en consecuencia, conocer cuáles han sido las lecciones aprendidas durante el año 2020, reconociendo de qué maneras han podido influir políticas inclusivas ya implantadas en las organizaciones para afrontar esta crisis.

En este trabajo ceñimos nuestra investigación al contexto iberoamericano, especialmente centrado en la realidad de las organizaciones en el contexto español en los últimos diez años y, particularmente, en el actual escenario covid y poscovid.

Los términos de búsqueda empleados, en documentos tanto en inglés como en español, según pertinencia y agrupados en bloques temáticos, han sido los siguientes:

- Conceptualización: diversidad, gestión humana, gestión de la diversidad, planes estratégicos.

- Elementos de la organización: clima, comunicación interna y externa, cultura, desempeño, recursos humanos, stakeholders, responsabilidad social corporativa.

- Gestión de la diversidad: antecedentes, barreras, beneficios, enfoques, evaluación, fases.

- Escenario covid en las organizaciones: barreras, beneficios, cambios, desafíos, retos.

Para ello hemos consultado el repositorio institucional de la Universidad Pontificia de Comillas, bases de datos online como Web of Science o Scopus, por ser referentes internacionales en publicaciones científicas, además de revistas académicas indexadas en estas bases de datos que tratan temáticas en torno a la gestión de las organizaciones, recursos humanos, responsabilidad social corporativa o gestión de la diversidad, como, por ejemplo, Revista Latina de Comunicación Social, Revista Iberoamericana de Desarrollo Humano y Social, Revista Digital de Capital Humano, Revista Dirección y Organización o Revista Internacional de Organizaciones. 
En la relativamente novedosa estrategia de gestión de la diversidad, se cuenta con numerosa y relevante información, estudios e informes por parte de las propias organizaciones y entidades como Fundación Once, Fundación CEPAIM, Club Excelencia en Gestión, Fundación ACCEM, Red Acoge, Observatorio de Conciliación, Corresponsabilidad y Diversidad de la Universidad Pontificia de Comillas o el Observatorio Europeo de la Gestión de la Diversidad.

Paralela a labores de revisión bibliográfica y por la excepcionalidad, irrupción e incertidumbre generadas por la crisis derivada de la covid-19, hemos realizado una entrevista estructurada a la Fundación Once, organización del entorno español capaz de dar claves y aportar matices acerca de la compleja realidad que nos ocupa, como, por ejemplo, necesarios cambios, beneficios y retos en el desarrollo de planes de gestión de la diversidad. Las preguntas realizadas han sido las siguientes:

- ¿Cómo ha afectado la crisis derivada de la covid-19 a sus planes internos relacionados con la gestión de la diversidad?

+ ¿En qué medida y cómo las políticas de diversidad existentes en su organización han sido y son útiles para gestionar mejor al personal durante esta crisis?

- Durante estos últimos meses de alarma, ¿han desarrollado alguna medida particular según las situaciones/características de cada empleado, como, por ejemplo, para las mujeres que actualmente están embarazadas, o para personas que tienen que cuidar a personas dependientes?

- ¿Cuáles cree usted que serán los principales desafíos y cambios para una gestión responsable de la diversidad a partir de la crisis generada por la covid-19?

- ¿Cuáles cree usted que han sido las principales lecciones aprendidas durante estos meses de alarma?

Como estudio de caso, hemos seleccionado a la Fundación Once por ser referente y autoridad en el Estado español en la realización de programas de integración laboral y de formación para personas con diversidad funcional desde 1998, la cual sirve como ejemplo en las buenas prácticas de gestión de una de las múltiples variables que componen el concepto de diversidad, motivo por el que ha sido reconocida con múltiples premios a su labor.

Otro criterio por el que hemos seleccionado el caso de Fundación Once se refiere a la amplia red de relaciones con las principales organizaciones de personas con discapacidad en España, donde destaca el Comité Español de Representantes de Personas con Discapacidad (CERMI), lo cual le permite formar parte de 
manera activa en la integración laboral y mejora de las condiciones de vida de los colectivos con los que trabaja.

La entrevista se ha desarrollado vía e-mail a causa de las medidas impuestas de confinamiento y seguridad. Escribimos al correo general de la Fundación Once, presentándonos como estudiantes de doctorado, especificando nuestro objeto de estudio y adjuntando nuestro guion cerrado de preguntas para responder. En cuanto al consentimiento informado, en nuestra presentación especificamos que la entrevista tiene fines de investigación y podrá ser utilizada en futuras publicaciones.

A la entrevista respondió uno de los técnicos de atención externa de la Fundación Once y experto en discapacidad, autonomía personal y atención a la dependencia. Su labor se refiere al establecimiento, comunicación y respuesta con/ entre los distintos agentes de la sociedad y stakeholders.

Para garantizar la confidencialidad de la persona entrevistada procedemos a mencionarla bajo el pseudónimo de Fundación Once en cada extracto de entrevista en que la citamos a lo largo de la discusión.

Como criterio de rigor se desarrolla una triangulación teórica acerca de la información obtenida en la entrevista, contrastándola con múltiples perspectivas y puntos de vista resultado de la consulta de publicaciones externas a la Fundación Once.

\section{Discusión}

La situación sociosanitaria causada por el virus de la covid-19 ha supuesto que prevalezcan medidas para preservar la salud y frenar el alto nivel de contagios que pueden perjudicar el normal desarrollo de la economía y el ámbito laboral y empresarial.

Medidas excepcionales, como, por ejemplo, el cese de actividad por un período determinado de tiempo, restricciones de movilidad, confinamiento domiciliario o la prohibición de reuniones de más de un determinado número de personas han supuesto un cambio de necesidades que afectan directamente a los objetivos organizacionales.

Estos cambios, tanto externos como internos, generados por dicha crisis, suponen «una transformación de características esenciales, que genera que el nuevo estado de cosas sea sustancialmente diferente al antiguo dentro de una organización» (García et alii, 2012:82).

Se habla de cambios episódicos, definidos como cambios ambientales externos y poco frecuentes que requieren de acciones intencionales y medidas excepcionales. 
Por tanto, para una adecuada adaptación a estos cambios organizacionales, se requieren previsión, anticipación y capacidad reacción (Weick y Quinn, 1999, citados por García et alii, 2012).

Para comenzar, será necesario realizar un diagnóstico de la situación de la organización, con la detección de las necesidades de cambio surgidas, para así poder planificar e implementar las acciones necesarias. Finalmente, tras ejecutar las medidas adoptadas, se llevará a cabo el control de los cambios y medidas adoptadas con la fase de evaluación.

Las políticas y planes en gestión responsable de la diversidad en las organizaciones han resultado primordiales para la adaptación de manera exitosa a las nuevas necesidades y cambios, pues la diversidad en las organizaciones es una de las problemáticas por estudiar y uno de los más importantes retos para las organizaciones (Pin et alii, 2007).

Myrtha Casanova (2006), experta en el tema, asegura que las organizaciones capaces de integrar e incluir la diversidad como centro en sus políticas, planes y ética podrán superar con éxito los futuros y diversos escenarios.

Dicha estrategia corporativa, centrada en integrar y potenciar las diferentes diversidades por razón de cultura, religión, edad, idioma u orientaciones sexuales, puede traducirse en «beneficios económicos y sociales dentro del marco de la Responsabilidad Social Corporativa» (Casanova, 2006: 1).

Para elaborar un plan de gestión de la diversidad, primero será necesario realizar un diagnóstico ajustado a las problemáticas y necesidades locales que ayude a comprender los contextos y poder así elaborar iniciativas apropiadas y efectivas (CIPD, Chartered Institute of Personnel and Development, 2019). Será necesario conocer:

- La legislación local.

- La cultura localः cuál es el punto de partida, cómo de abierto e inclusivo es el contexto local, ¿la organización se enmarca en una sociedad culturalmente abierta y legitima las diversidades?

- Cuestiones sociales locales: ¿cuáles son las problemáticas y colectivos con los que la sociedad está más concienciada y sensibilizada? Por ejemplo, algunos países priorizan problemáticas con respecto a la migración, o con respecto a las desigualdades socioeconómicas..., cada sociedad puede poner el foco en ciertas desigualdades, dejando otras en segundo plano.

Bien es cierto que, a menudo, las organizaciones desarrollan políticas y planes responsables no solo por la conciencia social y la voluntad proactiva, pero en otras ocasiones se dan por motivos legalistas, centrados en cumplir con la normativa o 
como estrategia de marketing en busca de mejorar la buena imagen de la marca, afianzando a los clientes (Porres, 2010).

Aunque los enfoques en la implementación de la diversidad puedan ser diferentes, todos conllevan la igualdad de oportunidades y la integración de colectivos desfavorecidos y minorías sociales en la vida laboral, mejorando sus condiciones personales y de trabajo (Raya y Martínez, 2009).

Pero, como avisa José Ramón Pin et alii (2007), la gestión de la diversidad que se hace solamente por cumplir normativas y legislación corre el riesgo de que el tratamiento de la diversidad sea solo superficial, percibiendo las medidas como una imposición, lo que hará perder su potencial.

Sea cual sea el enfoque $y / o$ motivo de su implementación, para que dicha gestión de la diversidad en las organizaciones funcione adecuadamente, el experto Gonzalo Sánchez Gardey (2021) señala la «función crucial» de las fuerzas internas, la alta dirección y los departamentos de recursos humanos para, por un lado, minimizar posibles consecuencias negativas de la diversidad, las cuales exploramos más adelante, y, por el otro lado, para «aprovechar al máximo sus beneficios potenciales» (p. 17).

Se deben tener en cuenta tres cuestiones importantes en la gestión de la diversidad. En primer lugar, su eficacia; en segundo lugar, su valor ético, donde se sitúa a las organizaciones como entidades sociales impulsando la inclusión social de diferentes colectivos de la sociedad y ofreciendo oportunidades de desarrollo. En tercer lugar, el autor señala la inevitabilidad de gestionar una diversidad cada vez más presente en las sociedades globalizadas, donde aparecen el envejecimiento de la población, la inmigración, la incorporación de la mujer al mundo laboral, el respeto a los derechos humanos o la visión inclusiva de las personas con discapacidad (Pin, 2007).

La gestión de la diversidad, como estrategia responsable de gestión de las personas en las organizaciones, se desarrolla desde los departamentos de recursos humanos, para los cuales, sin duda, integrar la diversidad es un reto a veces difícil de cumplir, pues afloran diferencias entre las personas, creándose tensiones y problemas en la relación y dificultades en la comunicación organizacional (Raya y Martínez, 2009).

Por tanto, para implantar exitosamente un modelo de gestión de la diversidad desde recursos humanos, el autor Gonzalo Sánchez Gardey considera que se deben considerar seis principios básicos (p.7):

- Énfasis en las competencias de comunicación.

+ Concienciación y sensibilización.

+ Liderazgo. 
- Gestión de la diversidad como un sistema de aprendizaje.

- Fomento de la interdependencia.

$+\quad$ Estructura de grupo.

En cuanto al primer principio básico, orientado a enfatizar las competencias comunicativas, será necesario el establecimiento de una fluida comunicación interna y externa, la cual ayude a fortalecer la cultura organizacional, y, por consiguiente, al afianzamiento de la identidad corporativa, capaz de crear correspondencia en/con el entorno, generando sentimiento de pertenencia en los empleados y en los stakebolders implicados, quienes son conocedores del valor añadido que sus características o peculiaridades aportan al desempeño del trabajo.

Relativo al segundo principio básico que Sánchez Gardey menciona como la cuestión «de concienciación y sensibilización» (p. 7), resulta de vital importancia recordar, y especialmente durante el actual período de crisis, la necesidad de prestar atención al hecho de «que no se vulnere ningún derecho fundamental» (Fundación Once).

La importancia en la defensa de los derechos humanos no siempre es reconocida en el seno de las organizaciones. Dicha cuestión resulta fundamental tanto para afianzar el sentido de pertenencia de los empleados como para mantener una corresponsabilidad que se establece con la sociedad en la que se insertan.

El tercer principio básico en el adecuado desarrollo de gestión de la diversidad se refiere al liderazgo. Estas medidas deben surgir de la alta dirección como un plan estratégico (Raya y Martínez, 2009).

Apostar por la diversidad en los planes estratégicos conecta directamente con el concepto de corresponsabilidad, es decir, con la generación de impacto social compartido. Se conecta directamente con el concepto de responsabilidad social corporativa (RSC) y con la idea del beneficio mutuo entre empresa, clientes, proveedores y sociedad.

Aunque no hay que confundir políticas de RSC con planes en gestión de la diversidad, pues, como indican la autora Adoración Alonso Rayas y el autor Gastón Martínez Heres (2009), ambas se diferencian en sus enfoques, ya que, aunque compartan puntos en común, como el respeto a los derechos humanos, la no discriminación o la mejora de la imagen y la reputación de la organización con la finalidad de obtener mayor competitividad, ambas difieren en el destinatario de las iniciativas.

La RSC se centra en la relación con el exterior y los stakeholders o las preocupaciones sociales y medioambientales. Sin embargo, la gestión de la diversidad apunta más a la cuenta de resultados: «[... [u trabajador que recibe un trato 
digno estará más comprometido con su tarea y aportará más valor a la empresa» (Rayas y Martínez, 2009: 59).

Por tanto, las principales diferencias entre RSC y gestión de la diversidad las encontramos en lo siguiente:

+ Los procesos de comunicación e innovación internas, a los que está enfocada la gestión de la diversidad, pero no las políticas de RSC.

- La relación con clientes y proveedores, pues, «a diferencia de la RSC, la gestión de la diversidad tiene como objetivo prioritario el acceso a nuevos mercados» (p. 59).

- La evaluación de resultados, pues la gestión de la diversidad, a diferencia de la RSC, está orientada a mejorar los resultados de la organización en cuanto a «reducción de costes, incremento de la productividad o acceso a nuevos mercados» (p.59).

Por último, centrando la discusión en el cuarto y el quinto de los principios básicos señalados por Gonzalo Sánchez Gardey necesarios para implantar exitosamente los modelos de gestión en diversidad, encontramos la importancia del fomento de la interdependencia y potenciación de la estructura de equipos de trabajo diversos, los cuales desarrollan proyectos innovadores y creativos.

Cuando el capital humano y los equipos son heterogéneos y diversos se potenciarán sus capacidades en tareas como recogida de información, toma de decisiones o solución de problemas. Personas con diferentes culturas, orientaciones y capacitaciones tendrán múltiples interpretaciones de la realidad y serán capaces de hacer un análisis más profundo de las cuestiones que tratar, lo que desencadena:

Mayor eficacia en la toma de decisiones y soluciones, de mayor calidad, a mayor velocidad, además de la creatividad y la novedad de las soluciones que se plantean [...]. Los grupos diversos tienen patrones de conocimiento y experiencia personal y laboral diversos y diferentes, lo que genera distintos puntos de vista, de formular el problema o la búsqueda de soluciones (Gardey, 2021: 9).

Integrar capital humano diverso en las plantillas aporta diferentes modos de ver $y$ hacer, con diversas perspectivas y diferentes visiones sobre el mundo que permiten obtener un amplio abanico de consideraciones y toma de decisiones, de manera que la empresa más heterogénea en trabajo interno se convertirá en más competitiva, lo que a su vez permitirá la apertura a nuevos mercados y clientes (Rodríguez, 2021).

Por tanto, la implementación de planes y estrategias socialmente responsables e inclusivas conlleva el cumplimiento del sexto principio básico establecido por 
Sánchez Gardey, es decir, desarrollar la gestión de la diversidad como un sistema de aprendizaje, orientado a los procesos.

Reorientando la discusión hacia los posibles beneficios, además de los ya mencionados, como resultado de la implementación de planes en gestión de la diversidad, destacamos las mejoras internas y de competitividad que estos conllevan, lo que genera crecimiento y mayores beneficios, además de una mayor creatividad a la hora de encontrar alternativas y soluciones ante problemas y retos, como los que sin duda han surgido en el actual escenario de incertidumbre y crisis sociosanitaria (Velasco, 2020).

En este sentido, la entidad Red Acoge, en el informe Índice de la gestión de la diversidad y la inclusión (2017), señala diferentes grupos de beneficios, distinguiendo entre internos de la propia organización, como el aumento del rendimiento, del desempeño, de la motivación de las personas o de la atracción y retención de talento, y beneficios externos, como mejora de la imagen y reputación de la organización, ventaja competitiva o mayor satisfacción de clientes y/o socios.

En dicho informe, Red Acoge demuestra los beneficios arriba mencionados, resultado de la implementación y evaluación de estrategias y medidas en torno a la gestión responsable de la diversidad en las organizaciones a través del desarrollo y posterior análisis de cuestionarios y entrevistas en los que participaron empleados de 28 empresas del Estado español que actúan en diferentes sectores de actividad, como la industria, la construcción, el comercio o los servicios, entre otros, de las que el 54\% de las personas encuestadas ocupan cargos en el área del capital humano, el 7\% de las personas encuestadas ocupan cargos específicos en gestión de la diversidad y el $18 \%$ son personas con cargos de dirección o gerencia (p. 15).

Sin duda, además de los beneficios mencionados, las organizaciones buscan una rentabilidad en todo establecimiento de estrategias y planes de acción que apuesten por la diversidad e inclusión. En este sentido, como apunta Fundación Once, dichos planes ya implementados antes de la crisis derivada de la covid-19 «han sido efectivos desde el minuto uno».

Es momento de que las organizaciones pongan a prueba su capacidad para responder a esta emergencia y demuestren esa responsabilidad social, igualdad y gestión de la diversidad con la que se han comprometido en sus páginas web (Velasco, 2020).

Ante el beneficio económico que la organización obtiene como resultado de estas mejoras, el ya referido estudio Índice IED de Red Acoge (2017) apunta a beneficios en los mercados de capital, es decir, en la atracción de inversores que comparten los mismos valores, beneficios en los productos aumentando la capacidad de respuesta ante las variadas necesidades de la clientela, beneficios en los 
mercados laborales, en la calidad y disponibilidad del personal laboral, además de beneficios derivados de subvenciones e incentivos de las Administraciones públicas.

Además, Red Acoge también apunta a la posibilidad de crear una positiva reputación de la organización de cara a los stakeholders, así como la obtención de una mayor ventaja competitiva.

A pesar de los beneficios correlacionados con la implementación de planes que apuestan por la integración de la diversidad, no se puede obviar la inevitable pérdida o afectación de servicios en esta crisis sociosanitaria.

Por ello, también es necesario señalar algunos argumentos que apuntan a posibles aspectos negativos y limitantes que puede conllevar una gestión responsable de la diversidad en las organizaciones.

Para algunos autores como Thomas Kochan (2002) y Miguel Ángel Sastre Castillo (2006), la gestión de la diversidad en las organizaciones carece de pruebas y evidencias probadas, además, argumentan que su aplicación no repercute en los resultados. Un posible efecto negativo de la diversidad puede ser un menor nivel de integración de equipos diversos frente a equipos de iguales.

Desde esta perspectiva, se apunta que la gestión de la diversidad no debe ser tomada como un modelo de aplicación universal ya que en determinadas tareas o actividades es recomendable la creación de equipos iguales. Esta perspectiva entiende que la búsqueda de heterogeneidad en los perfiles de los equipos resta vitalidad o dinamismo y pérdida de competitividad.

Por el contrario, la Escuela de Organización Industrial (EOI) opina que dichas perspectivas van en contra de una gestión responsable de la diversidad. Resultan obsoletas, pues conllevan la rigidez y la falta de implicación con la gestión de las personas en las organizaciones (Raya y Martínez, 2009).

En las sociedades actuales aparecen una enorme diversidad y heterogeneidad entre los agentes que las conforman. Las entidades deben adaptarse a dichos cambios, integrándolos y gestionándolos adecuadamente.

Por tanto, si se entiende como necesaria la implementación de planes y medidas en gestión de la diversidad en las organizaciones, también se deben tener en cuenta posibles barreras en su implementación a fin de anticipar las soluciones.

Como principal barrera se encuentra la adversidad al cambio por parte de los empleados, pues gestionar la diversidad implica moverlos de su zona de confort (Red Acoge, 2017).

Como segunda barrera encontramos las dificultades para vencer los estereotipos y prejuicios sobre las distintas variables de diversidad. Para vencerlos será necesario un trabajo continuo en formación, explicando qué es, para qué sirve, 
cuáles son sus beneficios, y convencer así al conjunto de la organización de los beneficios de gestionar la diversidad (Red Acoge, 2017).

Encontramos una tercera barrera, relacionada con la excepcional situación de pandemia, en el texto Los nueve desafíos que afronta la dirección de comunicación tras la COVID (2020) de José Antonio Velasco.

Se habla de la paralización y perjuicios en los servicios de proximidad, de comunicación o de la propia interacción con grupos externos. Estos se han visto mermados durante esta crisis sanitaria, tanto en la imposibilidad de realizar atención presencial durante los confinamientos, en las dificultades técnicas de reunirse por videoconferencia, como en la imprevisibilidad e incertidumbre que dificultan el cierre de citas previas con los distintos stakebolders.

Fundación Once nos habla de los inevitables perjuicios sufridos en concreto con las relaciones de proximidad con los agentes externos habituales, clientes o proveedores: «Ha afectado. Mi trabajo consiste en proporcionar información a las $\mathrm{PcD}$, y actualmente ese servicio de proximidad se ha visto mermado; presencial, teletrabajo, citas previas, etc.».

José Antonio continúa con la afirmación de que las acciones orientadas al fortalecimiento de los cauces de relación interna y con los grupos de interés, además del establecimiento de una comunicación rigurosa y oportuna, han sido elementos clave para abordar el nuevo escenario, pues actúan como escudo protector frente a la desesperanza, el pesimismo, la desmotivación o la desinformación.

El autor también señala a la cooperación entre los distintos departamentos de la organización como cuestión fundamental, lo que ha permitido responder rápida y eficazmente a las necesidades surgidas de las restricciones de movilidad, el cierre temporal de la actividad o la caída de las ventas.

Para la rápida respuesta ante problemas sobrevenidos, la comunicación interna y externa frecuente y de calidad resulta imprescindible para conseguir los objetivos de la organización, así como para establecer relaciones con otras organizaciones del entorno. La comunicación, como base del trabajo colaborativo, «se fortalece gracias a la construcción de vínculos, emociones y experiencias; las personas como seres sociales se mueven desde sus experiencias, tramas y contextos» (Apolo et alii, 2017:523).

En este sentido, el autor Gonzalo Sánchez Gardey (2021) presenta posibles efectos, tanto negativos como positivos, que pueden darse en la cooperación y el intercambio de información en equipos y stakeholders diversos y heterogéneos, sumando las dificultades e incertidumbre derivadas de la pandemia por la covid.

Como posible efecto negativo en la comunicación con empleados diversos, se tenderá a reducir la frecuencia de la comunicación interna, pues las minorías 
apenas alzan la voz y discrepan de las opiniones mayoritarias. De este modo se distorsiona el circuito de comunicación emisor/mensaje/receptor.

Esta reducción en la frecuencia de comunicación de equipos diversos puede generar una ausencia de retroalimentación y discusión en las propuestas de trabajo. Se genera miedo a opinar, perdiéndose así un funcionamiento adecuado del equipo en la búsqueda de mejoras y en la optimización de las tareas.

Para dichos problemas en la comunicación de organizaciones diversas, Sánchez Gardey propone el establecimiento de cauces de comunicación internos con un carácter formal que permitan reducir la interpretación del mensaje, al convertirse este en claro y preciso.

Por otro lado, el autor también señala efectos positivos en relación, sobre todo, con el exterior y la relación con los distintos stakeholders implicados.

Por ejemplo, la diversidad del equipo permite que cada uno se comunique con colectivos diferentes, llegando así a un público mayor y aumentando las redes de contacto, lo que mejorará la reputación y visión externa sobre la organización diversa, pues serán mejor percibidas y con una imagen más favorable.

Además de la comunicación, la situación actual de pandemia obliga a una transformación digital necesaria en las organizaciones, capaces así de adaptarse al nuevo escenario de trabajo en remoto desde casa como consecuencia de las restricciones de movilidad.

Ante este desafío, José Antonio Velasco recomienda hacer una digitalización lo más inclusiva posible. Esto conlleva una continua formación para aquellos empleados que no estén preparados, así como la adaptación de equipos según necesidades derivadas de diversidades funcionales.

El necesario desarrollo tecnológico y la transformación digital serán capaces de adaptarse a las nuevas necesidades, tanto de la plantilla y el trabajo remoto como con los clientes/consumidores (Velasco, 2020).

Para desarrollar de manera exitosa los necesarios cambios mencionados, se hace necesaria la planificación y sistematización de protocolos en la definición, comunicación e implementación de una estructurada ética organizacional que muestre su misión, valores y objetivos de forma clara y concisa, así como aquellas prácticas y conductas inaceptables (Velasco, 2020).

Tras discutir sobre los cambios y las necesidades en las organizaciones surgidas a raíz del escenario covid-19, así como los beneficios que suponen los planes en gestión de la diversidad, nos centramos en las lecciones ya aprendidas.

La asociación El Club Excelencia en Gestión, organizadora de los premios de Buenas Prácticas y del Sello EFQM, ha presentado el decálogo online Lecciones de gestión aprendidas de la covid-19 (2020), donde enumeran puntos de mejora 
para una rápida y eficaz respuesta a las situaciones de emergencia derivadas de la covid-19:

+ Priorizar el cuidado de la salud de los empleados.

- Anticipación y adaptación ante situaciones de emergencia.

- Establecer planes, estrategias y propósitos claros.

+ Desarrollar un liderazgo compartido y cultura organizacional sólida.

- Establecer buena comunicación que genera confianza y tranquilidad.

- Confiar en los beneficios de la colaboración y cooperación interna y externa.

+ Apostar por la autogestión y colaboración virtual.

+ Apostar por la digitalización, tecnología e innovación.

+ Contribuir en una sociedad solidaria y altruista.

En este sentido, Fundación Once apuesta por el afianzamiento de la cultura y los valores compartidos, como «afinidad, empatía, sentido de responsabilidad o teletrabajo», que ayuden a afrontar las dificultades que puedan surgir en las organizaciones.

El compromiso por afianzar los valores compartidos de las organizaciones resulta cuestión clave, además de para Fundación Once, para Juan Manuel Velasco o para Iñigo Arizmendiarrieta, que, como director de Talengo México, da claves para seguir adelante a partir de la crisis surgida de la covid-19 y la incertidumbre que esta conlleva.

Iñigo reconoce que la comunicación y colaboración deberán establecerse como acciones fundamentales, pues el trabajo colaborativo y en equipo permite la adaptación e integración de las nuevas realidades y necesidades.

Para ello, no solo es necesario contar con profesionales experimentados en un área de conocimiento específica, sino que, además, estos deberían conocer otras áreas. Conocimientos adquiridos en una formación continua que permita una mayor flexibilidad ante los cambios rápidos y constantes. «Se habla de una formación en «equipos multidisciplinares, flexibles, proactivos, automotivados, ágiles y orientados a resultados»» (Arizmendiarrieta, 2020).

Por último, y atendiendo al documento Gestión de la diversidad de la entidad, elaborado por Fundación CEPAIM (2013), se enuncian retos a los que las organizaciones deben hacer frente, consecuencia de las dificultades surgidas durante el año 2020.

En primer lugar, CEPAIM da claves para superar con éxito el reto de dar a conocer y difundir en la organización el plan elaborado o por elaborar acerca de la gestión de la diversidad. 
En primer lugar, es fundamental conseguir el compromiso real por parte de todas las personas implicadas en la organización, desde la base de la plantilla hasta la dirección y gerencia, venciendo así posibles resistencias al cambio y reconociendo los beneficios que trae consigo un plan de gestión de la diversidad, mediante el estímulo de comunicación y de diálogo.

Para ello, CEPAIM propone el desarrollo de un enfoque reactivo, capaz de adaptarse y dar respuestas a situaciones heterogéneas y capaz de integrar políticas de igualdad de oportunidades como eje estratégico en las empresas, en el acceso al empleo, en las recomendaciones de buenas prácticas de trato o en la eliminación de la discriminación, no solo para cumplir la legislación vigente sino también como una cuestión ética y de DD. HH.

En segundo lugar, se necesita establecer las condiciones que permitan desarrollar el potencial intrínseco de la diversidad presente en nuestra organización, empoderando a todos los implicados.

Con la integración del potencial de cada individuo y respetando su propia diversidad, se pretende un reparto más equitativo de poder, superando relaciones tradicionales y jerárquicas que permitan equilibrar relaciones de desigualdad (CEPAIM, 2020).

En un tercer reto, se habla del establecimiento de una planificación completa y adecuada que permita poner en marcha el proceso de gestión de la diversidad e igualdad de oportunidades, estableciendo acciones y estrategias conscientes, en trabajo de proceso.

Se menciona la importancia de revisar la política y cultura de la organización, suprimiendo aquellas acciones y comportamientos que supongan actitudes discriminatorias, estereotipadas y prejuiciosas que cada empleado tiene y las cuales resultan un peligro para la adecuada gestión de la diversidad (CEPAIM, 2013: 164).

Por tanto, la gestión responsable de la diversidad conlleva contar con las personas, atendiendo a su individualidad, detectando y aprovechando sus potencialidades para ponerlas a trabajar, respetando, escuchando e integrándolas en la misión y visión de la empresa.

Por último, destaca la importancia de la evaluación de los planes y estrategias en gestión de la diversidad, a fin de medir e informar sobre el impacto de estos sobre diferentes dimensiones de la organización como beneficio económico, así como información no financiera, relativa al desempeño, clima organizacional, implicación o motivación de los empleados.

La evaluación y el seguimiento de las acciones implementadas permiten obtener mejoras que posibilitan la sostenibilidad y el crecimiento de la organización. Y como señala el índice I\&D de Red Acoge (2017), permite «constatar el carác- 
ter reactivo de las medidas puestas en marcha y la falta de proactividad o capacidad de anticipación y control sobre el vector diversidad» (p.45).

Actualmente en las organizaciones se encuentra una dificultad para medir y evaluar los resultados derivados de los planes estratégicos. «El potencial de la diversidad queda inexplorado, por lo que decae el número de iniciativas en relación con las políticas corporativas de gestión de la diversidad» (Red Acoge, 2017: 10); además, se da una carencia de datos que evalúen e informen sobre el impacto y beneficios obtenidos.

A pesar de la importancia de evaluar los planes implementados, solo el $54 \%$ de las 28 empresas analizadas por Red Acoge disponen de mecanismos y cauces de respuesta frente a los conflictos que puedan surgir en torno a la discriminación, pues «[1] evaluación se dirige más a medir el impacto en el clima laboral, que al diagnóstico, medición y seguimiento de las acciones contenidas en las políticas de diversidad» (p. 46).

En la medida en que la evaluación nos permita conocer el impacto y los resultados derivados de los planes en gestión de la diversidad, la organización podrá redefinir los términos de implicación de cada empleado, redirigir recursos o esfuerzos y adecuar acciones según pertinencia.

Se recomienda que, como buena práctica para evaluar los datos y logros derivados de los planes en gestión de la diversidad, sean los propios empleados los que puedan identificar el nivel de tolerancia e importancia que cada contexto da a ciertas diversidades.

Además, deberían ser los empleados los encargados de monitorear la implantación y el progreso de los planes en gestión de la diversidad, así como evaluar su efectividad y mejoras (CIPD Chartered Institute of Personnel and Development, 2019).

Pero ¿se suelen medir el impacto y el retorno en las políticas en gestión en diversidad que se desarrollan desde los departamentos de recursos humanos?

En la investigación cuantitativa llevada a cabo por los autores Capapé, Susaeta et alii (2016), donde participaron 121 empresas, se concluyó que tan solo un $5,88 \%$ de las empresas encuestadas manifiestan tener herramientas que permitan evaluar el retorno e impacto resultado de las políticas realizadas desde los departamentos de recursos humanos.

Otra conclusión de este estudio es que, para la dirección general de las organizaciones participantes, evaluar y cuantificar datos en torno a políticas de formación y desarrollo interno resulta más relevante que evaluar políticas de reclutamiento y selección de la plantilla. 
Es decir, los procesos de atracción y retención de talento que permiten la contratación de empleados en función de sus méritos y capacidades mediante políticas de no discriminación no resultan prioritarios. Por el contrario, se le da mayor importancia a formar y desarrollar empleados ya contratados, reduciendo así la movilidad de estos y, por tanto, limitando la entrada de nuevo y diverso personal.

Más allá de los departamentos de recursos humanos, apuntamos a un importante indicador de evaluación de la gestión de la diversidad, tanto en las fases de diagnóstico, de implementación o de resultados. Este se refiere al alcance y la reacción emocional que los empleados y los distintos stakeholders tienen frente a las distintas diversidades, a fin de trabajarlas y gestionarlas para una adecuada integración.

Encarnación La Spina, del Instituto de Derechos Humanos Pedro Arrupe de la Universidad de Deusto, analiza el trasfondo emocional que subyace en la gestión de la diversidad en las organizaciones (2018).

Señala a la importancia que los estereotipos y prejuicios tienen contra la integración de la diversidad en los diversos ámbitos de la vida personal, familiar y laboral, pues, además de en nuestras relaciones sociales, también se reflejan en los empleados de una organización. Este recelo es fuente de conflicto frente a la idea de integrar estas diversidades en la entidad.

\section{Conclusiones}

Tras estudiar el caso de Fundación Once en relación con diferentes puntos de vista, concluimos que la implementación de planes de gestión de la diversidad en el caso estudiado aparece como planes estratégicos que surgen de la alta dirección y que, por tanto, están motivados por las propias fuerzas internas.

Dichos planes se enfocan hacia la mejora de la satisfacción de los empleados a través de la puesta en valor de su individualidad. A mayor satisfacción en el trabajo, mayor compromiso con la organización. «La gestión de la diversidad entiende el reconocimiento de las condiciones particulares de cada trabajador como un medio para alcanzar una mejora en los resultados de la empresa» (Rayas y Martínez, 2009: 58).

Para Fundación Once, trabajar la diversidad como forma de gestión responsable de las personas consigue que todos se sientan integrados y valorándolos por lo que son, convirtiendo así dichas diversidades y diferencias en oportunidad de crecimiento y mejora.

Dichos planes y estrategias socialmente responsables, por la consecución de igualdad entre hombres y mujeres y por la gestión e integración de las múltiples variables que el concepto de diversidad conlleva, se encuadran en la perspectiva 
de la gestión humana, que desde los departamentos de recursos humanos vienen desarrollando desde la segunda mitad del siglo xx (González, 2007).

Esta gestión humanista desarrollada desde Fundación Once se centra en el respeto por la dignidad humana y «el reconocimiento de la libertad, la autonomía de las personas» (González, 2007: 46), sin olvidar el necesario establecimiento de relaciones laborales más interpersonales y horizontales entre distintos cargos, en un proceso por entender las diversidades como potencial valor añadido a las capacidades profesionales.

Por otro lado, apuntamos, de manera general, como la crisis derivada de la covid-19 ha traído cambios radicales y excepcionales tanto a nivel ambiental externo como a nivel interno en las organizaciones a cota mundial donde se incluye la Fundación Once. Dicha excepcionalidad ha requerido de adaptaciones para superar con éxito las necesidades y los cambios surgidos. Se requieren previsión, anticipación y capacidad reacción.

Teniendo en cuenta la necesaria implementación de planes y medidas en la gestión responsable de la diversidad en las organizaciones en el actual escenario de crisis derivado de la covid-19, proponemos las siguientes acciones necesarias para su supervivencia:

- En primer lugar, para obtener los potenciales beneficios que conlleva gestionar la diversidad en las organizaciones, se aconseja su implementación planificada y estratégica, de manera transversal, a todos los niveles y cultura organizacional. Es necesaria la incorporación de la gestión de la diversidad como parte del modelo de negocio y en los ejes estratégicos de la compañía.

- Por tanto, para una adecuada adaptación a los cambios organizacionales, requieren previsión, anticipación y capacidad reacción a las nuevas situaciones. Es necesario realizar el diagnóstico de la situación actual, detectando las necesidades de cambio, para así poder planificar e implementar las acciones necesarias y adaptadas a la realidad de la organización, pues no existe una diversidad standard. Finalmente, la fase de evaluación permitirá llevar a cabo el control de los cambios y medidas adoptadas.

- Puesto que la diversidad es un concepto amplio que engloba diferentes variables y perfiles, en ocasiones visibles, como género, edad, diversidad funcional, etnia o cultura, y, en otras ocasiones, no visibles a simple vista, como el nivel educativo, la capacitación profesional o las habilidades personales, se recomienda una visión global e integradora en la organización en la búsqueda por equilibrar las distintas variables de la diversidad. 
- Resulta fundamental involucrar a todos los miembros de la comunidad en el proceso. Primero, las personas, atendiendo al sentido de pertenencia, integración y vinculación tanto de los empleados como de los agentes externos que permitan afianzar las relaciones de corresponsabilidad, confianza y relación positiva. Es necesario convertir los planes en gestión de la diversidad en objetivo estratégico que afecte a todos los escalafones de la empresa, desde la formación, la sensibilización y la contratación.

- Esto incluye definir, comunicar e implementar una estructurada ética organizacional, que muestre en su misión valores y objetivos de forma clara y concisa, así como aquellas prácticas y conductas inaceptables. La asimilación de los planes en diversidad por parte de todos los miembros de la organización se vuelve imprescindible, por eso es importante que la información y comunicación desde los niveles directivos y líderes sea compartida, clara y comprometida, que ayude a los empleados a comprender las decisiones y medidas tomadas.

- Mantener la defensa y respeto por los derechos fundamentales, bajo los que se sustentan valores colectivos como afinidad, empatía o sentido de responsabilidad, sirve como estrategia para afrontar las dificultades que puedan surgir, así como para afianzar políticas inclusivas que apuesten por una gestión corresponsable de la diversidad.

- Se vuelven fundamentales la atención y el cuidado de la dimensión emocional de las personas que forman parte de la organización.

- En la época actual se hace necesario desarrollar una digitalización inclusiva ante el inevitable avance tecnológico. Esto incluye adaptación de tecnologías y equipos tangibles a las necesidades particulares de cada empleado o la formación de aquellas personas que no cuentan con herramientas y conocimientos para desarrollar su trabajo eficazmente.

- Es necesario que el compromiso de la organización en atención a la diversidad incluya fases de análisis y evaluación de resultados, que permitan medir su impacto, así como la inclusión de mejoras. Se necesita hacer retrospectiva, evaluar, y si fuese necesario, reordenar prioridades, valores y medidas, haciendo política corporativa.

- Por último, hay que recordar que atender a estas políticas y planes inclusivos basados en el respeto por la diversidad resulta clave para una adaptación exitosa a los cambios derivados de la crisis de la covid-19. 


\section{Referencias}

ACCEM (2020). Empresas que Suman. Reto 2030. Proyecto Diven. Disponible en: <https://divem.accem.es/wp-content/uploads/2020/06/empresas_ que_suman_reto_2030.pdf $>$

Apolo, D., Báez, V., Pauker, L. y Pasquel, G. (2017). «Gestión de Comunicación Corporativa: consideraciones para el abordaje de su estudio y práctica». Revista Latina de Comunicación Social, 72, 521-539. Disponible en: <http:// www.revistalatinacs.org/072paper/1177/27es.html>

Arizmendiarrieta, I. (2020). Profesionales y empresas exitosas en el panorama COVID/Post-COVID. Talengo México. Disponible en: https://www.talengo.com/profesionales-y-empresas-exitosas-en-el-panorama-covid-post-covid/

Capapé, J., Susaeta, L., Pin, J. R., Danvila, I. y Suárez, E. (2016). «¿Se mide el retorno de la inversión en las políticas de Recursos Humanos? Un análisis en España». Innovar, 26 (59), 91-100. Disponible en: <https://doi. org/10.15446/innovar.v26n59.54365>

Casanova, M. (2006). La gestión de la diversidad. Unidad de conocimiento. Factorhumano.org. Disponible en: < http://fundacionellacuria.org/wp-content/ uploads/2009/07/Gestion-de-la-diversidad.pdf $>$

CIPD (2019). Diversity management that works. An evidence-based view. Chartered Institute of Personnel and Development. Disponible en: <https://www. cipd.co.uk/Images/7926-diversity-and-inclusion-report-revised_tcm1865334.pdf>

Comisión Europea (2020). LGBTIQ Equality Strategy 2020- 2025. Union of Equality. Communication from the Commission to the European Parliament, the Council, the European Economic and Social Committee and the Committee of the Regions.

Club Excelencia en Gestión (2020). Lecciones de Gestión aprendidas de la covid-19. Disponible en: <https://www.clubexcelencia.org/system/files/ knowledge/doc/lecciones-aprendidas-covid19-resumen-v2.pdf>

Fundación CEPAIM (2013). Gestión de la diversidad en entornos profesionales. Cuaderno para la formación. Disponible en: <http://cepaim.org/wpcontent/uploads/2015/02/2_edicion_Gestión-de-la-diversidad-Modulo01-CEPAIM-WEB.pdf>

Fundación CEPAIM (29 de marzo de 2020). Gestión de la diversidad. Responsabilidad social y gestión de la diversidad en tiempos de coronavirus. Cepaim.org. Disponible en: <http://cepaim.org/gestion-de-la-diversidad-responsabilidad-social-y-gestion-de-la-diversidad-en-tiempos-de-coronavirus/> 
García, M., Arias, F. y Gómez, P. (2013). «Relación entre comunicación y cambio organizacional en trabajadores de una empresa del sector terciario». Diversitas: Perspectivas Psicológicas, 9 (1), 81-95.

González, L. (2007). «Humanismo y gestión humana: una perspectiva de interpretación para el trabajo social aplicado al campo laboral». Revista Eleuthera, 1, 42-63. Universidad de Caldas. Disponible en: <https://www.redalyc. org/articulo.oa?id=585961830004>

Kochan, T., Bezrukova, K., Ely, R., Jackson, S., Joshi, A., Jehn, K., Leonard, J., Levine, D. y Thomas, D. (2003). «The effects of diversity on business performance: Report of the Diver- sity Research Network». Human Resource Management, spring, 42 (1), 3-21.

La Spina, E. (2018). «El trasfondo emocional en la gestión de la diversidad: una revisión crítica». Derechos y libertades, 38 (2), 271-297. Disponible en: <https://doi.org/10.14679/1063>

Observatorio de Conciliación, Corresponsabilidad y Diversidad (17 y 18 de noviembre de 2020). II Jornadas de Conciliación y Corresponsabilidad: los desafíos de la igualdad, la diversidad y la rentabilidad. Universidad Pontificia de Comillas, Madrid.

Pin, J. R., García, P. y Gallifa, A. (2007). Libro blanco sobre la gestión de la diversidad en las empresas españolas: retos, oportunidades y buenas prácticas. IESE Business School. Disponible en: <https://media.iese.edu/research/pdfs/ ESTUDIO-95.pdf>

Porres, M. (2010). «La discriminación laboral y la gestión de la diversidad de los recursos humanos» Revista Técnico Laboral, 32 (126), 513-568. Disponible en: <http://hdl.handle.net/2445/44668>

Raya, A. y Martínez, G. (2009). La Gestión de la Diversidad en las empresas españolas. Área de Conocimiento y Documentación de la Escuela de negocios EOI Escuela de Organización Industrial.

Red Acoge (2017) «Primer índice de la Gestión de la diversidad y la inclusión». Disponible en: <https $/ /$ redacoge.org/red-acoge-presenta-el-primer-informe-sobre-diversidad-e-inclusion-en-las-empresas-espanolas/ >

Rodríguez, L. F. (2021). «Retos y tendencias en la gestión de las personas». Revista digital Capital Humano. Disponible en: <https://capitalhumano. wolterskluwer.es/ch/2021/02/01/retos-y-tendencias-en-la-gestion-de-personas $>$

Sánchez, G. (2021). La diversidad en los grupos de trabajo: efectos potenciales y posibilidades de gestión. Instituto Europeo para la Gestión de la Diversidad. [Preprint] Disponible en: <http //www.iegd.org/spanish800/adjuntos/gestiondiversidadunivcadiz.pdf $>$ 
Sastre Castillo, M. (2004). «La gestión de la diversidad en la empresa». Dirección Estratégica, 11, 20-25.

Velasco, J. M. (2020). «Los nueve desafíos que afronta la dirección de comunicación tras la COVID». Disponible en: <https://www.fabulasdecomunicacion.es/2020/12/14/los-nuevos-desafios-que-afronta-la-direccion-decomunicacion-tras-la-covid/>

Weick, K. y Quinn, R. (1999). «Organizational change and development». Annual Review of Psychology, 50, 361- 386. 